\title{
Proposal for a standard micromagnetic problem: Spin wave dispersion in a magnonic waveguide
}

\author{
G. Venkat, D. Kumar, M. Franchin, O. Dmytriiev, M. Mruczkiewicz, H. Fangohr, A. Barman, M. Krawczyk, and
}

A. Prabhakar

\begin{abstract}
We propose a standard micromagnetic problem, of a nanostripe of permalloy. We study the magnetization dynamics and describe methods of extracting features from simulations. Spin wave dispersion curves, relating frequency and wave vector, are obtained for wave propagation in different directions relative to the axis of the waveguide and the external applied field. Simulation results using both finite element (Nmag) and finite difference (OOMMF) methods are compared against analytic results, for different ranges of the wave vector.
\end{abstract}

\section{INTRODUCTION}

There have been steady improvements in computational micromagnetics in recent years, both in techniques as well as in the use of graphical processing units (GPUs) [1]-[4]. These are complemented by efforts to compute the magnetization dynamics in various kinds of magnonic crystals and waveguides, of different geometries and made of different materials [5]-[7]. The dispersion relation, $\omega(k)$, provides valuable insights into the characteristics of propagating spin waves (SWs), and aids in our pursuit of building functional devices around magnonic waveguides [8]-[10]. Dispersion relations have traditionally been obtained using experimental means, which often means multiple experimental runs and associated costs and delays. Analytic solutions must rely on approximations that simplify the details of an experiment. Computational methods offer a compromise between the two, although agreement with analytic solutions can become elusive. This becomes evident in some of the figures in this article, where we observe good agreement in the dispersion relation for the fundamental propagating mode, but gradual disagreement as we study higher order modes. Our primary goal is to define a standard problem and provide sufficient numerical and analytic support to establish the dynamics in a simple magnonic waveguide.

The Landau-Lifshitz-Gilbert (LLG) [11] equation is the governing differential equation that describes the magnetization dynamics. There are many packages and methods used to solve the LLG equation. Among them are the Object Oriented Micromagnetic Framework (OOMMF) [12], LLG [13], Micromagus [14] and Nmag [15]. We rely on the finite difference

G. Venkat and A. Prabhakar are with the Dept. of Electrical Engineering, Indian Institute of Technology - Madras, Chennai 600036, India (email: anilpr@ee.iitm.ac.in)

D. Kumar, A. Barman are with the Dept. of Material Sciences, S. N. Bose National Centre for Basic Sciences, Salt Lake City, Kolkata - 700098, India.

M. Franchin and H. Fangohr are with the School of Engineering Sciences, University of Southampton, Southampton, SO17 1BJ, United Kingdom.

O. Dmytriiev is with the School of Physics, University of Exeter, Exeter, EX4 4QL, United Kingdom.

M. Mruczkiewicz and M. Krawczyk are with the Surface Physics Division, Faculty of Physics, Adam Mickiewicz University, Umultowska 85, 61-614 Poznań, Poland. method (FDM) adopted by OOMMF and the finite element method (FEM) used in Nmag. The latter is more suitable for geometries with irregular edges [16]. However, the computation overhead and management of resources become major issues in FEM simulations. To compare different numerical solvers, the Micromagnetic Modeling Activity Group ( $\mu \mathrm{Mag}$ ) publishes standard problems for micromagnetism [17]-[19]. A more recent addition included the effects of spin transfer torque [20]. However, there has thus far been no standard problem that includes the calculation of the spin wave dispersion of a magnonic waveguide. We believe that specifying a standard problem will promote the use of micromagnetic simulations and assist in the design of experiments to observe magnetization dynamics.

\section{PROBLEM SELECTION}

For a standard problem, we require that (a) different simulation tools can produce the same initial magnetization configuration, (b) the excitation field perturbs the magnetization sufficiently to excite multiple spin wave modes (c) computational times are reasonable, and (d) the results can be verified, preferably compared against analytic expressions. We define a problem that satisfies these criteria.

- The problem is separated into two sub problems which are tackled with two different simulations. The first deals with obtaining the initial magnetization for the configuration by applying the bias field along a direction suitable for the configuration. The resulting relaxed magnetization state obtained is used as initial magnetization for the following step.

- We apply an excitation that is varying as a sinc pulse in both time and space. Various other excitations were tried out but the one mentioned above led to the optimum dispersion curves. The sinc concentrates the SW power in a window in the frequency domain, and the dispersion curve obtained is prominent in detail. In particular, the excitation would launch all the modes in the frequency range of interest. The same logic was applied in determining the wave vector range of the excitation. The excitation should also be continuous in time, i.e., the amplitude of the excitation is determined at each time-step of the solver and not at any pre-determined time instants. Both OOMMF and NMAG had provisions to do this.

- In the relaxation simulation, the damping parameter $\alpha$, in the LLG equation, is kept high for faster convergence. At the same time, the convergence criterion is made stiffer to avoid a loss of accuracy. For the second simulation, 
we disable the convergence criterion (on the time rate of change of magnetization) and stopped the simulation after a given length of time.

- We save magnetization data (as a function of space and time), as provided by the packages used for the simulation. This data is then used for the purpose of obtaining the dispersion relation data, by using the Discrete Fourier transform (DFT) [21].

\section{PROBLEM DEFINITION}

The proposed geometry for the problem is that of a nanostripe of permalloy $(1000 \times 50 \times 1) \mathrm{nm}^{3}$, shown in Fig. 1 . By

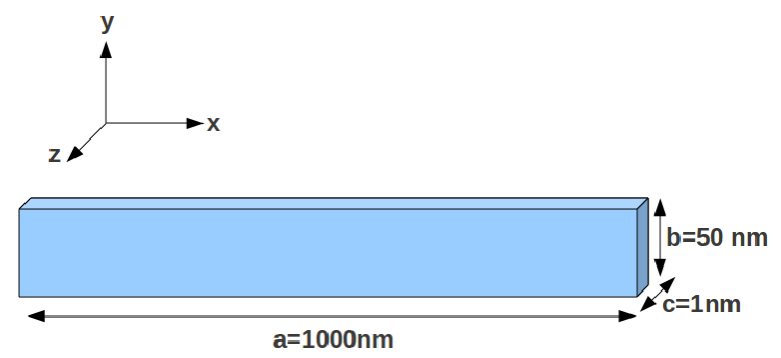

Figure 1. The geometry of the nano stripe.

choosing a cuboid, we ensure that the FDM method, used in OOMMF, does not introduce errors due to irregular edges.

- The length of the stripe was chosen to be long enough to make the notion of dispersion, $\omega(\mathrm{k})$, meaningful and to minimize edge effects.

- The width was chosen to make the possibility of standing wave occurrence, in the cross-section, realistic.

- The thickness was chosen to be small enough to safely assume that the dynamics are uniform along this direction.

Further specifications of the problem are given in Table I. Parameter values chosen are typical for permalloy, with the exception of the damping parameter $\alpha$ [22].

\begin{tabular}{|l|l|}
\hline Parameter & Value \\
\hline \hline Saturation Magnetization $\left(M_{\mathrm{s}}\right)$ & $8.6 \times 10^{5} \mathrm{~A} / \mathrm{m}$ \\
\hline Exchange Constant $(A)$ & $1.3 \times 10^{-11} \mathrm{~J} / \mathrm{m}$ \\
\hline Anisotropy Constant $(K)$ & 0 \\
\hline Gyromagnetic $\operatorname{ratio}(\gamma)$ & $1.7 \times 10^{9} \mathrm{~Hz} / \mathrm{T}$ \\
\hline Damping coefficient $(\alpha)$ & 0 \\
\hline DC bias field $\left(H_{0}\right)$ & $10.1 \mathrm{kOe}$ \\
\hline
\end{tabular}

Table I

FieldS AND CONSTANTS USED IN THE SIMULATION.

\section{RESULTS AND DISCUSSIONS}

In Fig. 2 we provide a schematic depiction of the different SW excitation configurations.

1) In the backward volume (BV) wave configuration, the external bias field $\left(\mathbf{H}_{0}\right)$ is along the length of the stripe and parallel to the wave vector $(\mathbf{k})$.

2) In the forward volume (FV) wave configuration, $\mathbf{H}_{0}$ is normal to the plane of the stripe with $\mathbf{k}$ being in the plane of the stripe.
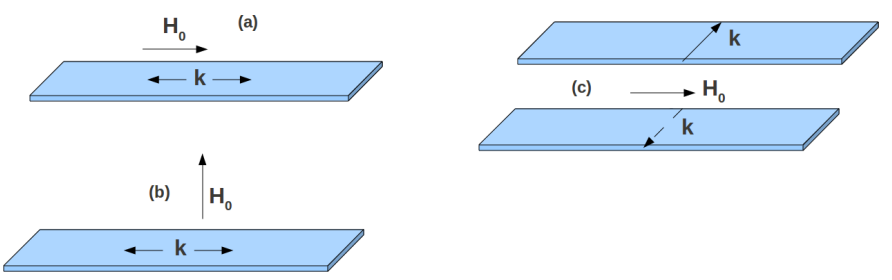

Figure 2. Excitation geometry for (a) backward volume, (b) forward volume and (c) surface spin waves.

3) Surface waves are also forward volume waves, i.e. the phase velocity $\left(v_{p}\right)$ and the group velocity $\left(v_{g}\right)$ are along the same direction. Surface waves are excited when both $\mathbf{H}_{0}$ and $\mathbf{k}$ are in the plane of the stripe, but are mutually perpendicular to each other.

A comparison with Fig. 1 suggests that the BV configuration is the most likely to be used in a magnonic device. However, for reasons of completeness, we will also present simulation results for the other two configurations.

\section{A. Meshing}

A few details about the statistics of the unit cell (in the case of OOMMF) and the mesh used (in the case of Nmag) are in order. A stringent condition to follow in the creation of the meshes is that the maximum mesh element size should not exceed the exchange length of the material. The exchange length for permalloy is around $5 \mathrm{~nm}$. This criterion is stressed upon so that the effect of exchange interactions is kept prominent.

Since OOMMF is a finite difference solver, it uses a regular grid and we set the cell size to $1 \mathrm{~nm} \times 2 \mathrm{~nm} \times 2 \mathrm{~nm}$, with the shortest edge along the thickness of the waveguide. It was also possible to simulate an extended stripe length by application of a periodic boundary condition, with the help of an OOMMF extension [23].

In the case of the Nmag, initially, the package NETGEN [24] (which uses the advancing front method) was used to create the mesh for the simulations. However, this did not meet the criteria above. Instead, we used a mesh created by decomposing the cuboidal body into cubes [20]. The mesh generated had 65025 volume elements, 45184 surface elements. The average mesh length was $2.21 \mathrm{~nm}$ while the maximum mesh length was $2.52 \mathrm{~nm}$.

\section{B. Excitation}

In order to excite SWs, an excitation pulse $h(x, y, t)$ was applied (along directions which differed according to the direction of $k$ for each of the three configurations). The excitation was a sinc pulse, with the mathematical form :

$$
h(x, y, t)=\Gamma \frac{\sin \left[k_{\mathrm{c}} x^{\prime}\right]}{k_{\mathrm{c}} x^{\prime}} \frac{\sin \left[k_{\mathrm{c}} y^{\prime}\right]}{k_{\mathrm{c}} y^{\prime}} \frac{\sin \left[2 \pi f_{\mathrm{c}} t^{\prime}\right]}{2 \pi f_{\mathrm{c}} t^{\prime}} .
$$

Defining $x^{\prime}=x-x_{0}, y^{\prime}=y-y_{0}$ and $t^{\prime}=t-t_{0}$, the excitation was offset by $t_{0}=50 \mathrm{ps}$ and was applied at the center of the stripe at $\left(x_{0}, y_{0}\right)=(500 \mathrm{~nm}, 25 \mathrm{~nm}) . k_{\mathrm{c}}$ is the cut-off value for the wave vector $k$, i.e., we excite waves with wave number 


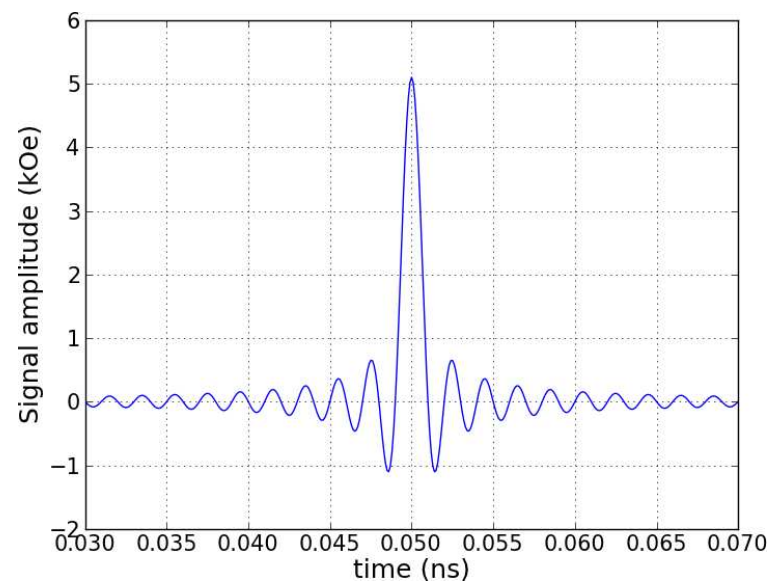

Figure 3. The applied excitation pulse, $h\left(500 \times 10^{-9}, 50 \times 10^{-9}, t\right)$, starts at $t=0$, has a maximum at $t=50 \mathrm{ps}$ and continues for the duration of the simulation.

$-k_{\mathrm{c}}<k_{x}<k_{\mathrm{c}}$. To obtain $N=250$ points in the dispersion curve, with $L=1 \mu \mathrm{m}$, we choose

$$
k_{\mathrm{c}}=\frac{N}{2} \Delta k=\frac{N}{2} \times \frac{2 \pi}{L}=2 \pi \times 0.1255 \times 10^{9} \mathrm{rad} / \mathrm{m} .
$$

$\Gamma=5.1 \mathrm{kOe}$ was the maximum amplitude of the excitation and $f_{\mathrm{c}}=500 \mathrm{GHz}$ was the cutoff frequency. A part of the excitation, is shown in Fig. 3.

\section{Simulation Methodology}

We first calculate the lowest energy state in the presence of an external bias field. The convergence criterion was set by a tolerance value for $\varepsilon$,

$$
\max \left\{\frac{1}{M_{\mathrm{S}}}\left|\frac{d \mathbf{M}_{i}}{d t}\right|\right\}_{i} \leq \varepsilon
$$

Nmag uses the ODE solver from Sundials [25]. The simulation was run thrice, with $\varepsilon$ being progressively tightened, and the absolute and relative tolerances on the time step $(\delta \tau)$ were also reduced each time. The values for $(\varepsilon, \delta \tau)$ used in the three simulations were $\left(1,10^{-5}\right),\left(0.1,10^{-6}\right)$ and $\left(0.01,10^{-7}\right)$, with the output of the first and second runs acting as input to the second and third runs, respectively. This step-wise relaxation allowed us to achieve faster convergence with an artificially inflated damping parameter, $\alpha=1$, without compromising the energy minimization procedure. A more systematic approach is described in [26].The hysteresis loop (shown in Fig. 4) of the stripe is obtained by applying the external bias field along $\hat{x}$ and $\hat{z}$, the easy (in the plane) and hard (out of the plane) axis of the stripe. This part of the simulation also allows us to

1) confirm that the chosen value of $H_{0}=10.1 \mathrm{kOe}$ is greater than the hard axis saturation field, and

2) save $\mathbf{m}(x, y, z, t)$, at the end of the simulation. The magnetization is saved cell-wise (for FDM) and sitewise (for FEM) and is used as $\mathbf{m}(x, y, z, 0)$ for the second simulation.

In the second part of the simulation, both $\mathbf{H}_{0}$ and $\mathbf{h}(x, y, t)$ are applied, $\alpha$ is set to zero and the simulation is allowed

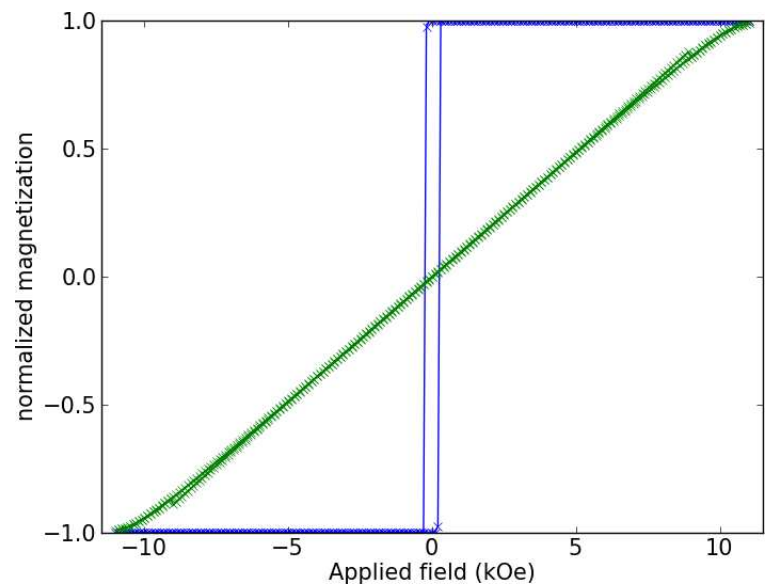

Figure 4. The hysteresis loop for the stripe, with the bias field applied along the easy $(x)$ and hard $(z)$ directions.

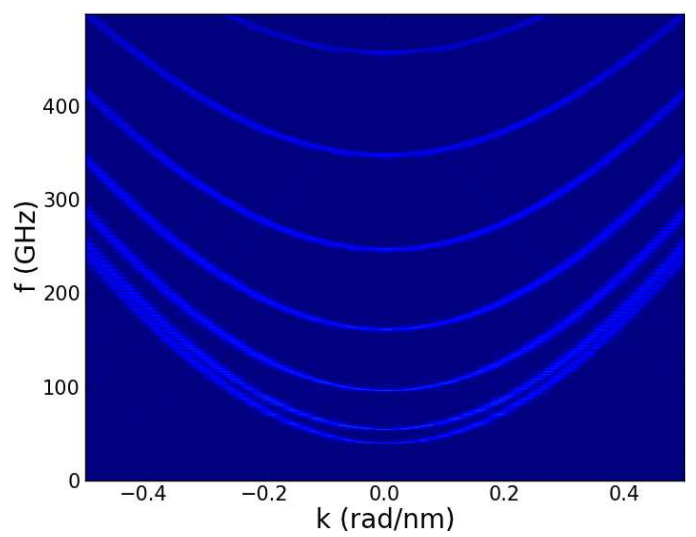

Figure 5. Surface plot of $m_{x}$, from (4), using data from an Nmag simulation. The curves depict the dispersion relation for BV spin waves in the nanostripe.

to relax till $5 \mathrm{~ns}$. The integration time step is computed using a second order backward difference formula [25], and $\mathbf{m}(x, y, z, t)$ was saved every pico-second. Setting $\alpha$ to zero improved the quality of the dispersion curves.

To generate a dispersion curve, we must view a surface plot of the magnetization components e.g. $m_{x}\left(k_{x}, \omega\right)$. We define

$$
m_{x}\left(k_{x}, \omega\right)=\mathcal{F}_{2}\left[m_{x}\left(x, y_{0}, z_{0}, t\right)-m_{x}\left(x, y_{0}, z_{0}, 0\right)\right],
$$

where $\mathcal{F}_{2}$ is the two dimensional Fourier transform and $y_{0}$ and $z_{0}$ are at the center of the stripe. The resulting surface plot, from Nmag simulations, is shown in Fig. 5.

A useful analytic approximation for spin wave dispersion was derived by Kalinikos [27]. For the lowest order modes, the result (after taking exchange interactions into account) is [28]

$$
\omega^{2}= \begin{cases}\omega_{\mathrm{ex}} \cdot\left(\omega_{\mathrm{ex}}+\omega_{\mathrm{M}} \frac{1-e^{-k c}}{k c}\right) & \mathrm{BV} \\ \omega_{\mathrm{ex}} \cdot\left(\omega_{\mathrm{ex}}+\omega_{\mathrm{M}}\left(1-\frac{1-e^{-k c}}{k c}\right)\right) & \mathrm{FV} \\ \omega_{\mathrm{ex}} \cdot\left(\omega_{\mathrm{ex}}+\omega_{\mathrm{M}}\right)+\frac{\omega_{\mathrm{M}}^{2}}{4}\left(1-e^{-2 k c}\right) & \text { Surface }\end{cases}
$$

where

$$
\omega_{\mathrm{ex}}=\omega_{0}+\lambda_{\mathrm{ex}} \omega_{\mathrm{M}} k^{2},
$$




\begin{tabular}{|c|c|}
\hline Variables & Typical value \\
\hline \hline$\lambda_{\mathrm{ex}}=\frac{2 \gamma A}{M_{\mathrm{s}}}$ & $3.23 \times 10^{-17} \mathrm{~m}^{2}$ \\
\hline$\omega_{\mathrm{M}}=\gamma \mu_{0} M_{\mathrm{s}}$ & $2 \pi \times 28.13 \mathrm{Grad} / \mathrm{sec}$ \\
\hline
\end{tabular}

Table II

VARIABLES COMMONLY USED IN ANALYTIC EQUATIONS.
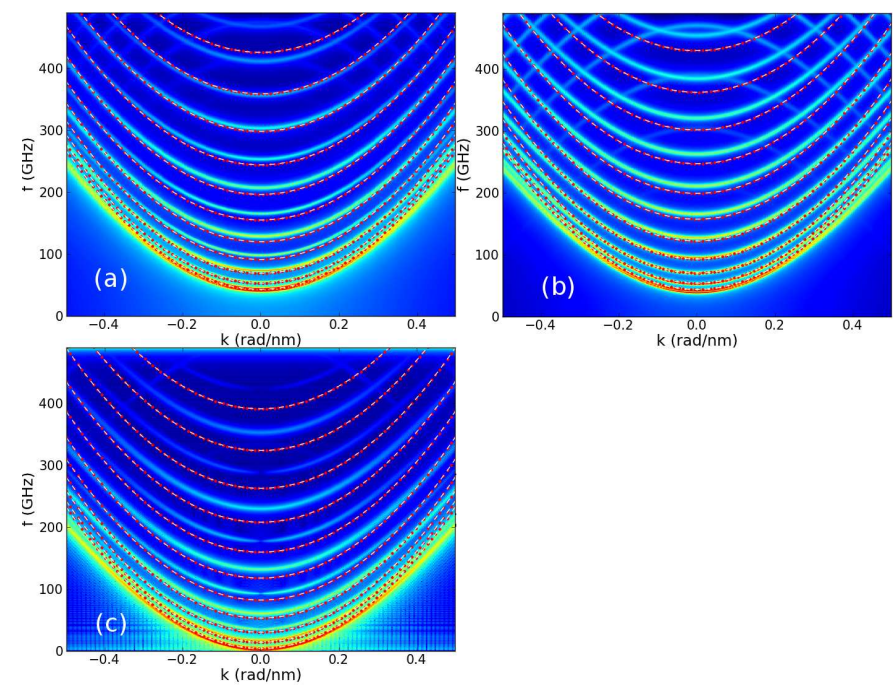

Figure 6. The dispersion curves, for (a) backward volume, (b) surface wave, and (c) forward volume configurations, obtained using OOMMF. The dots are the dispersion relations from the analytic models.

and

$$
\omega_{0}=\left\{\begin{array}{ll}
\gamma \mu_{0} H_{0} & \mathrm{BV} \text { and Surface } \\
\gamma \mu_{0}\left(H_{0}-M_{\mathrm{s}}\right) & \mathrm{FV}
\end{array} .\right.
$$

Note that $\lambda_{\mathrm{ex}}$ has units of $\mathrm{m}^{2}$. The remaining variables are defined in Table II. We assume that the demagnetization fields are negligible for the $\mathrm{BV}$ and surface wave cases, while $H_{\text {demag }}=-M_{\mathrm{S}}$ for the forward volume case.

Dynamic dipolar interactions can also influence the boundary conditions in the stripe [29]. This results in a special quantization condition along $y$

$$
k_{y}=\left(n_{y}+1\right) \frac{\pi}{b_{\mathrm{eff}}}, \quad n_{y}=0,1,2, \ldots
$$

for the different modes, where

$$
\begin{gathered}
b_{\mathrm{eff}}=b \frac{d}{d-2}, \\
d=\frac{2 \pi}{p\left[1+2 \ln \left(\frac{1}{p}\right)\right]}, \\
p=\frac{c}{b},
\end{gathered}
$$

and $k=\sqrt{k_{x}^{2}+k_{y}^{2}}$. (8) has been derived under the assumption that $c \ll b$ i.e. $p \ll 1$. In our case $p=0.02$.

The excitation signal in (1) is asymmetric about the $y=y_{0}$ line passing through the centre of the stripe. To excite both

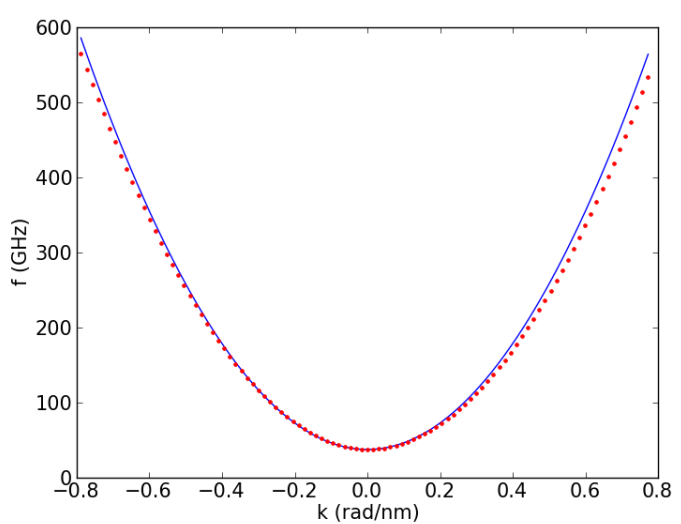

Figure 7. Optimal values of $\omega_{0}=2 \pi \times 26.07 \mathrm{GHz}$ and $\lambda_{\text {exch }}=2.96 \times$ $10^{-17} \mathrm{~m}^{2}$ were obtained by fitting (5) (dotted line) to (13). Restricting the fit to the region $\left|k_{x}\right|<0.1 \mathrm{rad} / \mathrm{nm}$ yielded a fit with $R^{2}=0.9949$.

even and odd modes, we modify the excitation to have the form

$$
h(x, y, t)=\Gamma \frac{\sin \left[k_{\mathrm{c}} x^{\prime}\right]}{k_{\mathrm{c}} x^{\prime}} \frac{\sin \left[2 \pi f_{\mathrm{c}} t^{\prime}\right]}{2 \pi f_{\mathrm{c}} t^{\prime}} \sum_{i=1}^{N} \sin \left[\frac{i \pi y}{y_{\max }}\right],
$$

where $y_{\max }=50 \mathrm{~nm}$ and $N=25$. This time we use OOMMF to generate the dispersion curves, shown in Fig. 6 . The analytic form of the first few modes, superimposed as dots, seems to agree well with the quadratic form of the dispersion curves from the simulations. For higher order modes we observe that theory predicts a higher value for $\omega\left(k_{x} \rightarrow 0\right)$ compared to what we see in the simulations. This difference is more pronounced in the case of BV waves. A second observation is that as $k \rightarrow k_{\mathrm{c}}$, there is a larger discrepancy between analytic and simulated values of $\omega\left(k_{x}\right)$. This is due to the proximity to the Brillouin boundary, along $k_{x}$, and can be reduced by reducing the micromagnetic cell size [30]. We also observe reflections of the dispersion curves about $f=f_{\mathrm{c}}$, which appear because we take the discrete Fourier transform on a finite length time series. The use of a periodic boundary condition, along the length, did not alter the the $\omega\left(k_{x}\right)$ curves.

\section{BACKWARD VOLUME WAVES}

The BV configuration is the most natural scheme for excitation of SWs in a nano-stripe, as $\mathbf{H}_{0} \| \mathbf{k}$, and both are along the length of the stripe. Hence, we use this configuration to establish the validity of the dispersion relations obtained from micromagnetic simulations vis-a-vis those obtained from analytic approximations for a thin film geometry.

To facilitate a detailed comparison with analytic models we extract the dispersion curve of the fundamental mode from the surface plot and fit it to a polynomial (in $\mathrm{rad} / \mathrm{s}$ ):

$$
\omega\left(k_{x}\right)=\left(5.54 \times 10^{-6}\right) k_{x}^{2}+2.36 \times 10^{11},
$$

where $k_{x}$ is the wave vector along the length shown in Fig. 7. As $k_{x} \rightarrow 0, \omega \rightarrow \sqrt{\omega_{0}\left(\omega_{0}+\omega_{\mathrm{M}}\right)} \approx 2 \pi \times 37.6 \mathrm{Grad} / \mathrm{sec}$, demonstrating a reasonable agreement between the analytic and simulation results for $\left|k_{x}\right| \leq 0.1 \mathrm{rad} / \mathrm{nm}$. 


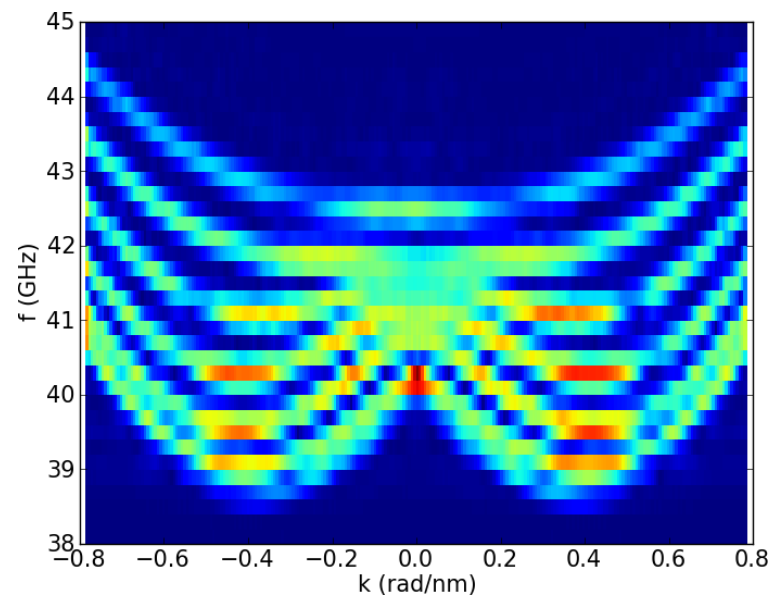

Figure 8. The dispersion curve exhibiting BV behavior, using Nmag, with $A=2.515 \times 10^{-13} \mathrm{~J} / \mathrm{m}$

It should be noted that (5) shows BV behaviour only for small values of $k_{x}$, where phase and group velocities have opposite signs. For $\left|k_{x}\right| \geq 2.8 \times 10^{6} \mathrm{rad} / \mathrm{m}$ the exchange term $\left(k_{x}^{2}\right)$ dominates the dipolar term. Increasing the length of the stripe to $10 \mu \mathrm{m}$ would give us enough resolution in $k_{x}$ to see $\mathrm{BV}$ behaviour, but would significantly increase the computational complexity. Instead, we changed the value of $A$ to $2.515 \times 10^{-13} \mathrm{~J} / \mathrm{m}$. Nmag simulations gave the dispersion curve shown in Fig. 8 where a Hanning window function [31]

$$
H(n)=\frac{1}{2}\left[1-\cos \left(\frac{2 \pi n}{N-1}\right)\right], \quad 0 \leq n \leq N-1
$$

with $N=5000$ was applied to smoothen the plot. The minimum is clearly visible at $k_{x, \min } \approx 4 \times 10^{8} \mathrm{rad} / \mathrm{nm}$.

When the exchange interactions are comparable to the dipolar interactions, we need to improve upon the approximations made in the derivation of (5). The dispersion relation for the $\mathrm{BV}$ configuration (with exchange interactions) is [32]

$$
\omega^{2}=\omega_{\mathrm{ex}}\left(\omega_{\mathrm{ex}}+\omega_{\mathrm{M}} \frac{k_{y}^{2}+k_{z}^{2}}{k_{x}^{2}+k_{y}^{2}+k_{z}^{2}}\right),
$$

where, for the odd modes (here we are considering the first mode), $k_{z}$ is solved for from the relation

$$
k_{x}=k_{z} \tan \left(k_{z} c\right)
$$

and $k_{y}=0$. Using the value of the reduced exchange constant, $A=2.515 \times 10^{-13} \mathrm{~J} / \mathrm{m}$, we plot the analytic forms of (5) and (15) in Fig. 9. (5) is also plotted with the additional quantization in (8) for $n_{y}=0$. A comparison with Fig. 8 suggests that we still have finite size effects that are not captured by the analytic approximations.

\section{SUMMARY}

As we begin designing magnonic waveguides, we must also find ways of simulating spin wave propagation in these nano-structures. The dispersion curves are integral to our understanding of spin wave propagation. By defining a standard problem around a nano-stripe geometry, we facilitate a comparison between different micromagnetic packages, and

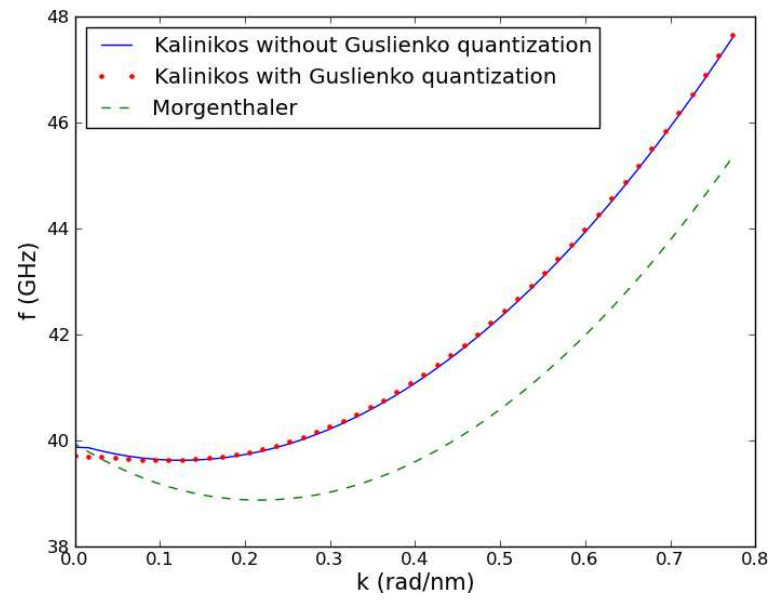

Figure 9. BV dispersion curves, from (5) without including (8), (5) after including (8), and (15). The first two coincide, except in the $k \rightarrow 0$ limit. Zero group velocity occurs at $k_{x, \min } \approx 1.3 \times 10^{8} \mathrm{rad} / \mathrm{nm}, 1.1 \times 10^{8} \mathrm{rad} / \mathrm{nm}$ and $2.5 \times 10^{8} \mathrm{rad} / \mathrm{nm}$ for the three cases, respectively.

also between finite difference and finite element solvers. This would be a starting point, before embarking on studies to understand the influence of irregular shapes or edge deformities on spin wave excitation and propagation.

We have shown reasonable agreement between $\omega(k)$ obtained from micromagnetic simulations, and $\omega(k)$ obtained from analytic approximations, for different configurations of SWs. This is evident especially for the lower order modes. The BV nature of the waves is exhibited only for small values of $k$ as the exchange term dominates for large $k$. The analytic equations do not appear to accurately capture the dispersion behaviour for large $k$. We have shown that it is possible to obtain more resolution, in the region $k \rightarrow 0$ without increasing the length of the stripe, by altering the value of the phenomenological exchange constant $(A)$.

Micromagnetic simulations are sensitive to factors such as the discretization in the geometry, the features of the excitation pulse (temporal and spatial variation, maximum amplitude), the damping constant, and post processing of the data [7], [26], [33]. While we have tried to systematize these parameters, we must still rely on practice and experience to obtain good dispersion curves.

\section{ACKNOWLEDGMENT}

The research leading to these results has received funding from the European Community's Seventh Framework Programme (FP7/2007-2013) and from the Department of Science and Technology, Government of India under the India-EU collaborative project DYNAMAG (grant number INT/EC/CMS (24/233552)). DK would like to acknowledge the financial support received from the CSIR - Senior Research Fellowship (File ID: 09/575/(0090)/2011 EMR-I)

\section{REFERENCES}

[1] S. Li, B. Livshitz, and V. Lomakin, "Graphics processing unit accelerated O(n) micromagnetic solver," IEEE Trans. Magn., vol. 46, pp. 2373 $2375,2010$. 
[2] A. Kakay, E. Westphal, and R. Hertel, "Speedup of FEM micromagnetic simulations with graphical processing units," IEEE Trans. Magn., vol. 46, pp. $2303-2306,2010$.

[3] O. Bottauscio and A. Manzin, "Efficiency of the geometric integration of Landau-Lifshitz-Gilbert equation based on Cayley transform," IEEE Trans. Magn., vol. 47, pp. $1154-1157,2011$.

[4] C. Abert, G. Selke, B. Kruger, and A. Drews, "A fast finite-difference method for micromagnetics using the magnetic scalar potential," IEEE Trans. Magn., vol. PP, p. 1, 2011.

[5] B. C. Choi et al., "Nonequilibrium magnetic domain structures as a function of speed of switching process in $\mathrm{Ni}_{80} \mathrm{Fe}_{20}$ thin-film element," IEEE Trans. Magn., vol. 43, pp. 2 -5, 2007.

[6] S.-K. Kim, "Micromagnetic computer simulations of spin waves in nanometre-scale patterned magnetic elements," J. Phys. D: App. Phys., vol. 43, p. 264004, 2010.

[7] M. Dvornik and V. V. Kruglyak, "Dispersion of collective magnonic modes in stacks of nanoscale magnetic elements," Phys. Rev. B, vol. 84, p. 140405, Oct 2011

[8] V. Kruglyak and R. Hicken, "Magnonics:experiment to prove the concept," J. Magn. Magn. Mater, vol. 306, pp. 191 - 194, 2006.

[9] A. Khitun, M. Bao, and K. Wang, "Spin wave magnetic nanofabric: A new approach to spin-based logic circuitry," IEEE Trans. Magn., vol. 44, pp. $2141-2152,2008$.

[10] S. Bance et al., "Micromagnetic calculation of spin wave propagation for magnetologic devices," J. Appl. Phys., vol. 103, p. 07E735, 2008.

[11] T. Gilbert, "A phenomenological theory of damping in ferromagnetic materials," IEEE Trans. Magn., vol. 40, pp. 3443 - 3449, 2004.

[12] M. Donahue and D. Porter, "OOMMF user's guide, version 1.0," National Institute of Standards and Technology, Gaithersburg, MD, Tech. Rep., 1999.

[13] M. R. Scheinfein, "LLG - micromagnetics simulator," 2008

[14] D. Berkov, "MicroMagus - software for micromagnetic simulation," 2008.

[15] T. Fischbacher et al., "A systematic approach to multiphysics extensions of finite-element-based micromagnetic simulations: Nmag," IEEE Trans. Magn., vol. 43, pp. 2896 -2898, 2007.

[16] O. Bottauscio, M. Chiampi, and A. Manzin, "A finite element procedure for dynamic micromagnetic computations," IEEE Trans. Magn., vol. 44, pp. $3149-3152,2008$.

[17] M. J. Donahue, D. G. Porter, R. D. McMichael, and J. Eicke, "Behavior of mu mag standard problem no. 2 in the small particle limit," vol. 87 , pp. 5520-5522, 2000

[18] "Finite element calculations on the single-domain limit of a ferromagnetic cube-a solution to $\mu$ MAG standard problem no. 3," Journal of Magnetism and Magnetic Materials, vol. 238, pp. 185 - 199, 2002.

[19] V. D. Tsiantos et al., "Stiffness analysis for the micromagnetic standard problem no. 4," Journal of Applied Physics, vol. 89, pp. 7600-7602, 2001.

[20] M. Najafi et al., "Proposal for a standard problem for micromagnetic simulations including spin-transfer torque," J. Appl. Phys., vol. 105, p. 113914, 2009.

[21] A. V. Oppenheim, R. W. Schafer, and J. R. Buck, Discrete-time signal processing, 2nd ed. Upper Saddle River, NJ, USA: Prentice-Hall, Inc. 1999.

[22] A. Hubert and R. Schafer, Magnetic Domains: The Analysis of Magnetic Microstructures. Berlin: Springer, 1998.

[23] K. M. Lebecki, M. J. Donahue, and M. W. Gutowski, "Periodic boundary conditions for demagnetization interactions in micromagnetic simulations," Journal of Physics D: Applied Physics, vol. 41, p. 175005, 2008.

[24] J. Schoberl, "NETGEN an advancing front 2d/3d-mesh generator based on abstract rules," Computing and Visualization in Science, vol. 1, pp. 41-52, 1997.

[25] A. C. Hindmarsh et al., "Sundials: Suite of nonlinear and differential/algebraic equation solvers," ACM Trans. Math. Softw., vol. 31, pp. 363-396, 2005

[26] O. Bottauscio and A. Manzin, "Critical aspects in micromagnetic computation of hysteresis loops of nanometer particles," IEEE Trans. Magn., vol. 45, pp. $5204-5207,2009$.

[27] B. Kalinikos, "Excitation of propagating spin waves in ferromagnetic films," IEE Proc., vol. 127, p. 4, 1980.

[28] D. D. Stancil and A. Prabhakar, Spin Waves Theory and Applications, 1st ed. New York: Springer, 2008.

[29] K. Y. Guslienko et al., "Effective dipolar boundary conditions for dynamic magnetization in thin magnetic stripes," Phys. Rev. B, p. 132402, 2002
[30] M. Dvornik, A. N. Kuchko, and V. V. Kruglyak, "Micromagnetic method of s-parameter characterization of magnonic devices," Journal of Applied Physics, vol. 109, no. 7, p. 07D350, 2011.

[31] D. Kumar, O. Dmytriiev, and A. Barman, "Numerical calculation of the dispersion of magnon waves in different kinds of magnonic crystals," in Proc. IEEE International Magnetics Conference (Intermag' 11), Taipei, Taiwan, 2011.

[32] F. Morgenthaler, "An overview of electromagnetic and spin angular momentum mechanical waves in ferrite media," Proc. IEEE, vol. 76, pp. $138-150,1988$.

[33] D. Kumar et al., "Numerical calculation of spin wave dispersions in magnetic nanostructures," Journal of Physics D: Applied Physics, vol. 45, no. 1, p. 015001, 2012. 